Research Round Up-Treatment Advances in Mental Health

Introduction

The last research round up provided you with a review of antimicrobial prescribing practices in patients being treated for SARS-CoV-2 either the use of these for COVID-19 itself or for detected secondary infection. This month we are going to look at some interesting and applicable advances in the area of treatment of bipolar disorder, depression and anxiety. Some very new research is examined, and implications reviewed and some of the other papers are in areas of interest to mental health practitioners and those in other fields who may come across mental health conditions during their normal practice.

\title{
Trial of Psilocybin versus Escitalopram for Depression
}

\section{https://www-nejm-org.salford.idm.oclc.org/doi/pdf/10.1056/NEJMoa2032994?articleTools=true}

This phase 2, double-blind, randomized, controlled trial involving patients with long-standing, moderate-to-severe major depressive disorder was conducted over a 6-week period and compared the traditionally used antidepressant drug escitalopram with psilocybin. Patients were assigned in a 1:1 ratio to receive two separate doses of $25 \mathrm{mg}$ of psilocybin 3 weeks apart plus 6 weeks of daily placebo (psilocybin group) or two separate doses of $1 \mathrm{mg}$ of psilocybin 3 weeks apart plus 6 weeks of daily oral escitalopram (escitalopram group); all the patients received psychological support. The trial was conducted by researchers at Imperial College London.

The psychedelic compounds and psilocybin occur naturally in the psychoactive psilocybe genus of mushrooms. As with other traditional psychedelic substances, the main effects of psilocybin occur through serotonin (5-HT2A) receptor agonism, which is part of a pathway implicated in depression. Psilocybin may have antidepressant properties, but direct comparisons between psilocybin and established treatments for depression were lacking prompting the authors to conduct this trial.

The primary outcome measure was the change from baseline in the score on the 16-item Quick Inventory of Depressive Symptomatology-Self-Report (QIDS-SR-16: scores range from 0 to 27, with higher scores indicating greater depression) at week 6 . There were 16 secondary outcomes measured, including QIDS-SR-16 response (defined as a reduction in score of $>50 \%$ ) and QIDS-SR-16 remission (defined as a score of $\leq 5$ ) at week 6 .

Men and women between the ages of 18 and 80 years were recruited formally through trial networks. In total of 59 patients were enrolled; 30 were assigned to the psilocybin group and 29 to the escitalopram group. Randomization (performed with the use of a random-number generator) was implemented by staff members who were not part of the research team. The mean scores on the QIDS-SR-16 at baseline were 14.5 in the psilocybin group and 16.4 in the escitalopram group. The changes in the scores from baseline to week 6 were -8.0 in the psilocybin group and -6.0 in the escitalopram group. A QIDS-SR-16 response occurred in $70 \%$ of the patients in the psilocybin group and in $48 \%$ of those in the escitalopram group. QIDS-SR-16 remission occurred in $57 \%$ and $28 \%$, respectively. Other secondary outcomes generally favoured psilocybin over escitalopram. The incidence of adverse events was similar in the trial groups.

Based on changes in scores, this trial did not show a significant difference in antidepressant effects between psilocybin and escitalopram in a selected group of patients. Secondary outcomes generally 
favoured psilocybin over escitalopram. The authors conclude from these that larger and longer trials are required to compare psilocybin with established antidepressants.

Carhart-Harris, R., Giribaldi, B., Watts, R., Baker-Jones, M., Murphy-Beiner, A., Murphy, R., Martell, J., Blemings, A., Erritzoe, D., \& Nutt, D.J. (2021) Trial of Psilocybin versus Escitalopram for depression New England Journal of Medicine: 384;15, 1402-1411

New Fathers' Perinatal Depression and Anxiety_Treatment Options: An Integrative Review https://journals.sagepub.com/doi/pdf/10.1177/1557988316669047

This review of the available literature around perinatal depression and anxiety in new fathers was conducted with the aim of summarising the current understanding of the condition and any treatment programs specifically designed for fathers with perinatal depression. Thirteen articles were included in this review after a systematised search process and inclusion criteria were employed. Areas reviewed included detection, diagnosis, prevalence and therapeutic interventions including talking and pharmacological approaches.

More than $10 \%$ of fathers experience depression and anxiety during the perinatal period, but paternal perinatal depression (PPND) and anxiety have received less attention than maternal perinatal mental health problems. The evidence presented suggested a lack of support and of specifically tailored treatment options for men having trouble adjusting to the transition to fatherhood. Of the limited options available, cognitive behavioural therapy, group work, and blended delivery programs, including e-support approaches appear to be most effective in helping fathers with perinatal depression and anxiety with antidepressant medication having a similar effect when used.

The authors conclude that further research is needed to address the adoption of specific models of care to encourage fathers' help-seeking behaviour. Inclusion of male-specific requirements into support and treatment options can improve the ability of services to engage new fathers. Psychotherapeutic intervention could assist men adjusting to the role of father, including male identity and role expectations.

O’Brien, A.P., McNeil, K.A., Fletcher, R., Conrad, A., Wilson, A.J., Jones, D., \& Chan, S.W. (2017) New Fathers' Perinatal Depression and Anxiety-Treatment Options: An Integrative Review Mental Health \& Wellbeing: Vol. 11(4) 863-876

Efficacy of cariprazine in bipolar I depression across patient characteristics: a post hoc analysis of pooled randomized, placebo-controlled studies

https://www.ncbi.nlm.nih.gov/pmc/articles/PMC7846289/pdf/icp-36-76.pdf

This RCT aimed to investigate the effects of the drug cariprazine in patients with bipolar disorder. The authors wanted to see if its efficacy varied dependant on patient demographics such as age, sex, race, $\mathrm{BMI}$, duration of illness, disease severity and whether patients had received prior medication for bipolar disorder. Cariprazine is a broad-spectrum dopamine antagonist/partial agonist (DAPA) drug, more commonly used in the UK in the treatment of psychosis such as schizophrenia. This review of 3 trials using cariprazine pooled data from all $3 \mathrm{RCT}$, placebo-controlled trials. The outcome measure used was the Montgomery-Åsberg Depression Rating Scale (MADRS). 
The mean difference in MADRS change from baseline was statistically significant for cariprazine 1.5$3 \mathrm{mg} / \mathrm{d}$ versus placebo in all patient subgroups analysed ( $\mathrm{P}<0.05$ all subgroups): demographic characteristics (age, sex, white or black race and obese/nonobese BMI)); episode characteristics (defined by current episode duration, number of previous manic/mixed and depressive episodes, and prior bipolar disorder medication use) and disease severity (groups above and below Clinical Global Impressions-Severity and MADRS cut off scores). Cariprazine $1.5-3 \mathrm{mg} / \mathrm{d}$ consistently improved depressive symptoms in all patient subgroups without regard to differences in baseline demographic and clinical characteristics, suggesting broad efficacy across a spectrum of patients with bipolar disorder.

The authors conclude therefore that, cariprazine $1.5-3 \mathrm{mg} /$ day consistently improved depressive symptoms in all patient subgroups without regard to differences in baseline demographic and clinical characteristics, suggesting broad efficacy among patients with bipolar depression.

Patela ,M., Jainb , R., Tohenc , M., Maleticd ,V., Earleya, W.R. \& Yathame, L.N. (2021) Efficacy of cariprazine in bipolar I depression across patient characteristics: a post hoc analysis of pooled randomized, placebo-controlled studies International Clinical Psychopharmacology: 36:76-83

\section{Conclusion}

Detection of mental health disorders and signposting to appropriate support services is every prescriber's responsibility. Advances in pharmacological interventions for mental health disorders continues to be an important area of research. Investigations of current medications and their use in novel indications often comes from effects seen in patients with comorbid conditions. New and experimental research into drugs which have been used recreationally and seen, anecdotally, to show some benefit is also an important area of investigation in the quest for new and effective treatments. Prescribers need to be aware of these advances, especially as many are reported in the national media, to aid discussions with patients and clients. 